Rakenteiden Mekaniikka (Journal of Structural Mechanics)

Vol. 50, No 3, 2017, pp. 349 - 352

https://rakenteidenmekaniikka.journal.fi/index

https://doi.org/10.23998/rm.65307

(c) The author(s) 2017.

Open access under CC BY-SA 4.0 license.

\title{
A model for magneto-elastic behaviour
}

Siavash Danaee, Reijo Kouhia ${ }^{1}$, Paavo Rasilo, Anouar Belahcen, Deepak Singh, and Matti Ristinmaa

Summary. In this paper, a coupled magnetoelastic model for isotropic ferromagnetic materials is presented. The constitutive equations are written on the basis of the total energy in which the right Cauchy-Green strain tensor and the Lagrangian form of the magnetic field strength are used as the basic state variables. It is also applied to ferromagnetic electric steel for which the material parameters are calibrated.

Key words: magnetostriction, electric steel, total energy function, Lagrangian description

Received 29 July 2017. Accepted 19 August 2017. Published online 21 August 2017.

\section{Introduction}

In this paper a finite strain model for isotropic magnetostriction is developed. The model is based on the formulation introduced by Dorfmann and Ogden [3, 4] utilizing the concept of "total energy function". Since further development towards anisotropic behaviour is looked for, the formulation is given by using the Lagrangian description.

\section{Constitutive equations}

In magnetoelastostatics the three basic magnetic variables are the magnetic field $\boldsymbol{H}$, the magnetic induction $\boldsymbol{B}$ and the magnetization $\boldsymbol{M}$. The fields $\boldsymbol{H}$ and $\boldsymbol{B}$ are considered as the primary fields and $\boldsymbol{M}$ only as an auxiliary field [5], which can be defined in terms of $\boldsymbol{H}$ and $\boldsymbol{B}$.

In electromagnetics it is customary to work with the Eulerian frame, so the field $\boldsymbol{H}$ and $\boldsymbol{B}$ are related to the current configuration. Since our future goal is to model anisotropic behaviour where the material orientation is important, a material description of motion is chosen. The Lagrangian forms of the primary magnetic fields are

$$
\boldsymbol{H}_{\mathrm{L}} \equiv \boldsymbol{F}^{T} \boldsymbol{H}, \quad \text { and } \quad \boldsymbol{B}_{\mathrm{L}} \equiv J \boldsymbol{F}^{-1} \boldsymbol{B},
$$

where $\boldsymbol{F}$ is the deformation gradient and $J=\operatorname{det} \boldsymbol{F}$. For further details see [3, 4].

\footnotetext{
${ }^{1}$ Corresponding author. reijo.kouhia@tut.fi
} 
Denoting the complementary form of the total energy function as $\Omega^{*}\left(\boldsymbol{F}, \boldsymbol{H}_{\mathrm{L}}\right)$, and using the standard Coleman-Noll procedure, the total stress $\boldsymbol{\tau}$ and the magnetic induction $\boldsymbol{B}$ can be obtained from equations

$$
\boldsymbol{\tau}=J^{-1} \boldsymbol{F} \frac{\partial \Omega^{*}}{\partial \boldsymbol{F}}, \quad \boldsymbol{B}=-J^{-1} \boldsymbol{F} \frac{\partial \Omega^{*}}{\partial \boldsymbol{H}_{\mathrm{L}}} .
$$

The function $\Omega^{*}\left(\boldsymbol{F}, \boldsymbol{H}_{\mathrm{L}}\right)$ is a partial Legendre transform of the total energy function $\Omega\left(\boldsymbol{F}, \boldsymbol{B}_{\mathrm{L}}\right)$, i.e.

$$
\Omega^{*}\left(\boldsymbol{F}, \boldsymbol{H}_{\mathrm{L}}\right)=\Omega\left(\boldsymbol{F}, \boldsymbol{B}_{\mathrm{L}}\right)-\boldsymbol{H}_{\mathrm{L}} \cdot \boldsymbol{B}_{\mathrm{L}} .
$$

The total energy function $\Omega$ is related to the Helmholtz free energy per unit mass $\psi$ as

$$
\Omega \equiv \rho_{0} \Phi+\frac{1}{2} \mu_{0}^{-1} J \boldsymbol{B} \cdot \boldsymbol{B}, \quad \text { where } \quad \Phi\left(\boldsymbol{F}, \boldsymbol{B}_{\mathrm{L}}\right) \equiv \psi\left(\boldsymbol{F}, J^{-1} \boldsymbol{F} \boldsymbol{B}_{\mathrm{L}}\right)
$$

in which $\rho_{0}, \mu_{0}$ are the density in the reference configuration and the magnetic permeability in vacuum, respectively.

To model isotropic magnetostriction, the energy is assumed to depend on the right Cauchy-Green deformation tensor $\boldsymbol{C}=\boldsymbol{F}^{T} \boldsymbol{F}$ and the Lagrangian magnetic field $\boldsymbol{H}_{\mathrm{L}}$. Integrity basis of a scalar function depending of a symmetric second order tensor and a vector consist of the following six invariants [6]:

$$
\begin{aligned}
I_{1}=\operatorname{tr} \boldsymbol{C}, I_{2}=\frac{1}{2}\left[(\operatorname{tr} \boldsymbol{C})^{2}-\operatorname{tr} \boldsymbol{C}^{2}\right], I_{3}=\operatorname{det} \boldsymbol{C}, I_{4}=\boldsymbol{H}_{\mathrm{L}} \cdot \boldsymbol{H}_{\mathrm{L}}, \\
I_{5}=\boldsymbol{H}_{\mathrm{L}} \cdot \boldsymbol{C} \boldsymbol{H}_{\mathrm{L}}, I_{6}=\boldsymbol{H}_{\mathrm{L}} \cdot \boldsymbol{C}^{2} \boldsymbol{H}_{\mathrm{L}} .
\end{aligned}
$$

From equations (2) expressions for the total stress tensor $\boldsymbol{\tau}$ and the magnetic induction vector $\boldsymbol{B}$ are found as

$$
\boldsymbol{\tau}=J^{-1} \boldsymbol{F} \sum_{k=1}^{6} \frac{\partial \Omega^{*}}{\partial I_{k}} \frac{\partial I_{k}}{\partial \boldsymbol{F}}, \quad \boldsymbol{B}=-J^{-1} \boldsymbol{F} \sum_{k=1}^{6} \frac{\partial \Omega^{*}}{\partial I_{k}} \frac{\partial I_{k}}{\partial \boldsymbol{H}_{\mathrm{L}}} .
$$

Evaluation of the derivatives $\partial \Omega^{*} / \partial \boldsymbol{F}$ and $\partial \Omega^{*} / \partial \boldsymbol{H}_{\mathrm{L}}$ gives

$$
\begin{aligned}
\boldsymbol{\tau}= & J^{-1}\left[2 \boldsymbol{b} \Omega_{1}^{*}+2\left(I_{1} \boldsymbol{b}-\boldsymbol{b}^{2}\right) \Omega_{2}^{*}+2 I_{3} \boldsymbol{I} \Omega_{3}^{*}+2 \boldsymbol{b} \boldsymbol{H} \otimes \boldsymbol{b} \boldsymbol{H} \Omega_{5}^{*}\right. \\
& \left.+2\left(\boldsymbol{b} \boldsymbol{H} \otimes \boldsymbol{b}^{2} \boldsymbol{H}+\boldsymbol{b}^{2} \boldsymbol{H} \otimes \boldsymbol{b} \boldsymbol{H}\right) \Omega_{6}^{*}\right] \\
\boldsymbol{B}= & -J^{-1}\left(2 \boldsymbol{b} \boldsymbol{H} \Omega_{4}^{*}+2 \boldsymbol{b}^{2} \boldsymbol{H} \Omega_{5}^{*}+2 \boldsymbol{b}^{3} \boldsymbol{H} \Omega_{6}^{*}\right),
\end{aligned}
$$

where $\boldsymbol{b}=\boldsymbol{F} \boldsymbol{F}^{T}$ is the left Cauchy-Green deformation tensor, the notation $\Omega_{i}^{*}$ denotes the derivative $\Omega_{i}^{*}=\partial \Omega^{*} / \partial I_{i}$ and $\otimes$ is the standard tensor product.

The chosen total energy functional is additively decomposed to a purely elastic part

$$
\Omega^{* \mathrm{e}}=\frac{1}{2} K\left[\frac{1}{2}\left(J^{2}-1\right)-\ln J\right]+\frac{1}{2} G(\operatorname{tr} \overline{\boldsymbol{C}}-3)
$$

and a coupled magneto-elastic part

$$
\begin{aligned}
\Omega^{* \mathrm{~m}}= & -\frac{1}{2} \mu_{0}\left(1-\alpha_{5}-\alpha_{6}\right) I_{4}-\alpha_{4} \mu_{0} h_{0} \ln \left[\cosh \left(f(x) \sqrt{I_{4}} / h_{0}\right)\right] \\
& -\frac{1}{2} \alpha_{5} \mu_{0} h_{0}^{2}\left(I_{5} / h_{0}^{2}\right)-\frac{1}{2} \alpha_{6} \mu_{0} h_{0}^{2}\left\{1+\beta_{1}\left[1-\exp \left(-\beta_{2} I_{4} / h_{0}^{2}\right)\right]\right\}\left(I_{6} / h_{0}^{2}\right),
\end{aligned}
$$

where the isochoric part of the right Cauchy-Green deformation tensor is denoted as $\overline{\boldsymbol{C}}=$ $J^{-2 / 3} \boldsymbol{C}$ and $K, G$ are the bulk and shear moduli, respectively. The function $f(x)$, where 
Table 1. Coefficients of the $f$-function.

\begin{tabular}{ccccccccc}
\hline$k_{f}$ & $k_{s}$ & $10^{4} \cdot d$ & $s^{-}$ & $s^{+}$ & $f_{\infty}^{-}$ & $f_{\infty}^{+}$ & $10^{4} \cdot x_{m}$ & $f_{m}$ \\
\hline 5378.499 & 4431.884 & 1.75633 & 1.6775 & 0.531416 & 0.22914 & 1.00759 & 1.089 & 2.4 \\
\hline
\end{tabular}

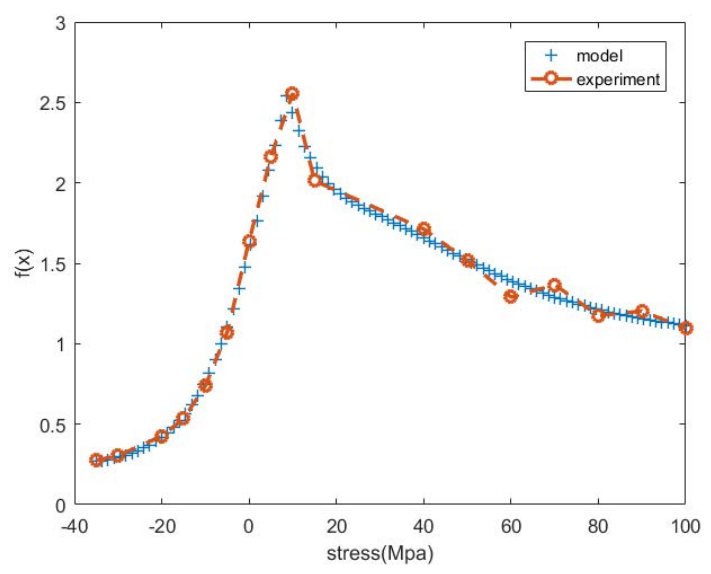

Figure 1. Measured and model forms of the $f$-function.

$x=\tilde{I}_{5}=\boldsymbol{H}_{\mathrm{L}} \cdot(\overline{\boldsymbol{C}}-\boldsymbol{I}) \cdot \boldsymbol{H}_{\mathrm{L}} / I_{4}$ is important in modelling the deformation dependency, i.e. the stress dependency of the initial magnetic permeability. For electric steel the following form is adopted based on experimental results

$$
f(x)=f_{\infty}(x)+\left(f_{\mathrm{m}}-f_{\infty}(x)\right) \exp \left\{-\sqrt{\left[\left(s(x) x-x_{\mathrm{m}}\right) / d\right]^{2}}\right\},
$$

and the infinity $f_{\infty}$ and slope function $s$ are of the form

$$
\begin{aligned}
f_{\infty}(x) & =\frac{1}{2}\left\{f_{\infty}^{-}+f_{\infty}^{+}+\left(f_{\infty}^{+}-f_{\infty}^{-}\right) \tanh \left[k_{f}\left(x-x_{\mathrm{m}}\right)\right]\right\} \\
s(x) & =\frac{1}{2}\left\{s^{-}+s^{+}+\left(s^{+}-s^{-}\right) \tanh \left[k_{s}\left(x-x_{\mathrm{m}}\right)\right]\right\} .
\end{aligned}
$$

Note that the function $f$ is insensitive to volumetric deformation. The behaviour of the initial magnetic permeability as a function of applied stress shows a peak and saturation both in tension and compression, therefore the rather complicated expression (11) contains nine parameters: $f_{\mathrm{m}}, x_{\mathrm{m}}, d, f_{\infty}^{-}, f_{\infty}^{+}, k_{f}, s^{+}, s^{-}$and $k_{s}$, which are estimated by standard least-squares fitting. The values obtained for the electric steel of grade Mxxx-50 $\mathrm{A}^{2}$ are shown in Table 1, see also Fig. 1. It should be noticed that the form of the function $f$ is material dependent.

The model parameters $\left(K, G, h_{0}, \alpha_{4}, \alpha_{5}, \alpha_{6}, \beta_{1}\right.$, and $\left.\beta_{2}\right)$ will be estimated from the experiments. It can be seen that depending on the signs and relative magnitudes of the coefficients $\alpha_{5}$ and $\alpha_{6}$, the following behavior can be obtained in the uniaxial case:

1. $\alpha_{5}<0, \alpha_{6}=0$ results in negative magnetostriction with saturation,

2. $\alpha_{5}>0, \alpha_{6}=0$ results in positive with $H$ monotonously increasing magnetostriction,

${ }^{2}$ Unfortunately the loss value of the studied material is not known. 
3. $\alpha_{5}=0, \alpha_{6}>0, \beta_{1}=0$ results in positive with $H$ monotonously increasing magnetostriction,

4. $\alpha_{5}>0, \alpha_{6}<0, \beta_{1}=0$ results in negative saturating magnetostriction,

5. $\alpha_{5}>0, \alpha_{6}<0, \beta_{1} \neq 0$, but $\left|\alpha_{6}\right|<\frac{1}{2} \alpha_{5}$ results in positive saturating magnetostriction,

6. $\alpha_{5}>0, \alpha_{6}<0, \beta_{1} \neq 0$, but $\left|\alpha_{6}\right|>\frac{1}{2} \alpha_{5}$ results in initially positive magnetostriction which however saturates to a negative value.

\section{Concluding remarks}

Modelling magnetoelastic behaviour is challenging due to the complexity of the coupled magnetomechanical problem. A Lagrangian formulation is adopted due to future expandability to an anisotropic case. Future study is still needed to explore the capabilities of the model.

Acknowledgements. The research leading to these results has received funding from the European Research Council under the European Union's Seventh Framework Programme (FP7/2007-2013) / ERC grant agreement $\mathrm{n}^{\circ} 339380$, from a strategic grant of Tampere University of Technology, from the Challenge Finland project "Clean Energy from Environment with Energy Harvesting" funded by Tekes the Finnish Funding Agency for Innovation, and from the Academy of Finland grants 274593 and 304112.

\section{References}

[1] L. Daniel and O. Hubert and N. Buiron and R. Billardon. Reversible magneto-elastic behaviour: A multiscale approach. Journal of the Mechanics and Physiscs of Solids, 56:1018-1042, 2008. https: //doi.org/10.1016/j.jmps.2007.06.003.

[2] L. Daniel. An analytical model for the effect of multiaxial stress on the magnetic susceptibility of ferromagnetic materials. IEEE Transactions on Magnetics, 49:(5) 2037-2040, 2013. https://doi. org/10.1109/TMAG. 2013.2239264.

[3] A. Dorfmann and R.W. Ogden. Nonlinear magnetoelastic deformations. Quarterly Journal of Mechanics and Applied Mathematics, 57:599-622, 2004. https://doi.org/10.1093/qjmam/57.4.599.

[4] A. Dorfmann and R.W. Ogden. Some problems in nonlinear magnetoelasticity. Zeitschrift für Angewandte Mathematik und Physik, 56:718-745, 2005. https://doi.org/10.1007/s00033-004-4066-z.

[5] A. Kovetz. Electromagnetic Theory. Oxford University Press, 2000.

[6] A.J.M. Spencer. Theory of invariants. In A.C. Eringen, editor, Continuum Physics, volume 1, pages 239-353. Academic Press, 1971. https://doi.org/10.1016/B978-0-12-240801-4.50008-X.

Siavash Danaee, Reijo Kouhia

Tampere University of Technology, Civil Engineering, P.O. Box 600, FI-33101 Tampere, Finland siavash.danaeefarborooshi@tut.fi, reijo.kouhia@tut.fi

Paavo Rasilo, Deepak Singh

Tampere University of Technology, Electrical Engineering, P.O. Box 692, FI-33101 Tampere, Finland paavo.rasilo@tut.fi, deepak.singh@tut.fi

Anouar Belahcen

Aalto University, Electrical Engineering, P.O. Box 13000, FI-00076 Aalto, Finland

anouar.helahcen@aalto.fi

Matti Ristinmaa

Lund University, Solid Mechanics, P.O. Box 118, SE-22100, Sweden

matti.ristinmaa@solid.lth.se 